\title{
Acute monoplegia secondary to herpes zoster infection: a case report
}

\author{
Meliha Kasapoglu Aksoy ${ }^{1}$, Lale Altan ${ }^{2}$, Sezin Solum ${ }^{1}$ \\ ${ }^{1}$ Department of Physical Medicine and Rehabilitation, University of Health Sciences, Bursa Yuksek Ihtisas Training and Research \\ Hospital, Bursa, Turkey \\ ${ }^{2}$ Department of Department of Physical Medicine and Rehabilitation, Uludag University School of Medicine, Bursa, Turkey
}

\begin{abstract}
Brachial plexopathy with motor involvement secondary to herpes zoster infection is very rare. Also known as shingles, herpes zoster occurs as a result of reactivation of varicella zoster virus in the dorsal root ganglia. We present a 69-year-old male patient with right upper extremity monoplegia secondary to herpes zoster infection. Physical examination showed scars from rash on his right antecubital fossa. Electroneuromyography showed a low amplitude motor response of the right median nerve, while having no sensation response at digits 1 and 3. Right ulnar and radial nerve sensation responses were lower in amplitude compared to the left side. Herpes zoster should be considered in the differential diagnosis of acute monoparesis. It is very important in avoiding unnecessary tests and treatments.
\end{abstract}

Eur Res J 2017;3(1):90-93

Keywords: Herpes zoster infection, acute monoplegia, brachial plexopathy

\section{Introduction}

Also known as shingles, herpes zoster occurs as a result of reactivation of varicella zoster virus in the dorsal root ganglia [1]. Postherpetic neuralgia is the most common complication of herpes zoster. The neurological complications of herpes zoster include aseptic meningitis, myelitis, encephalitis, GuillainBarre syndrome, development of stroke, and peripheral neuropathy $[1,2]$. Brachial plexopathy with motor involvement secondary to herpes zoster infection is very rare [2]. It is believed that motor involvement may develop due to dermatomes involved by extension of the inflammation from the dorsal root ganglia to the ventral roots. However, the exact pathology is unknown [3]. We present a case of right upper extremity monoplegia secondary to herpes zoster infection.

\section{Case Presentation}

A 69-year-old male patient presented to our clinic with a complaint of pain and weakness in the right upper extremity that started 3 months ago. It was a burning pain, and the patient described discomfort in daily activities when using his right upper extremity and right hand. He had no history of trauma. During 
anamnesis, he described a vesicular itchy rash on the antecubital fossa in the right arm, which started 3 days before the onset of pain and weakness. He had presented to our dermatology department for his complaint of rash, and received antiviral treatment. He had diagnosis of diabetes mellitus and hypertension in his history. He had no specific features in his family. Physical examination showed scars from rash on his right antecubital fossa (Figure 1).

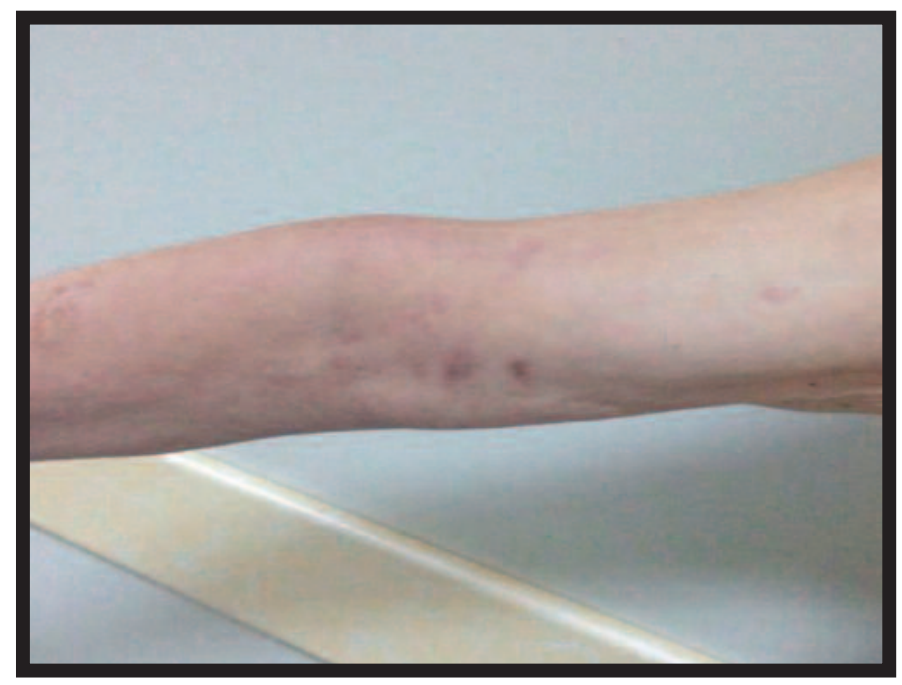

Figure 1. Appearance of scars from rash on right antecubital fossa.

There was no atrophy in the muscles of the right upper extremity (Figure 2). The cervical range of motion (ROM) was full active, and painless. No sign of spurling's was found. The passive and active ROM of the shoulder, elbow, wrist and fingers were full and painless.

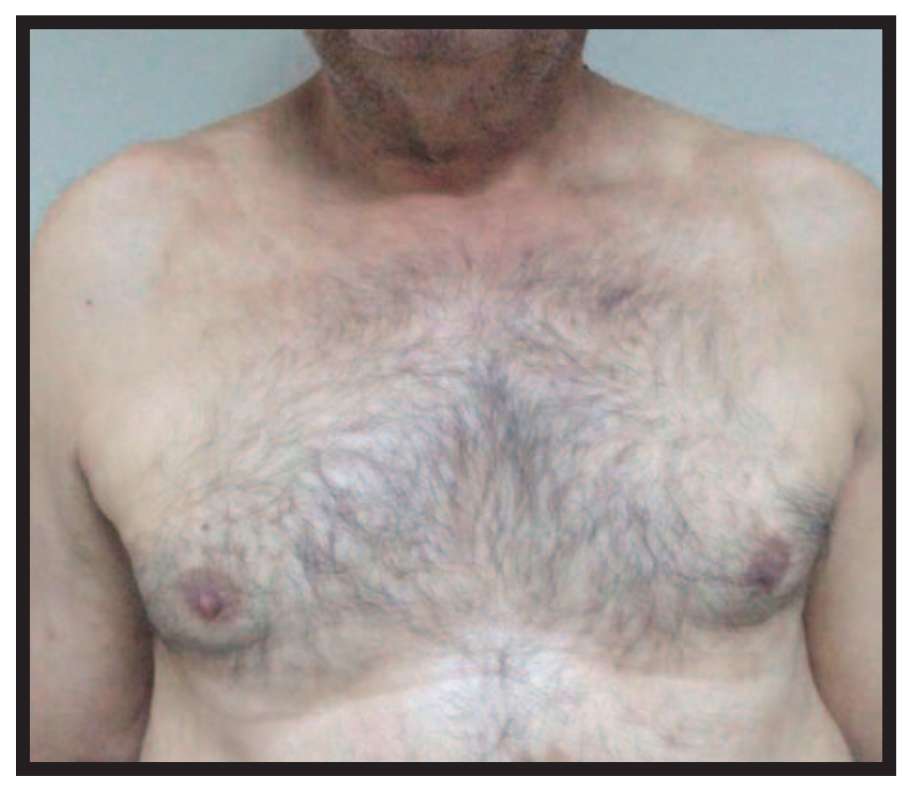

Figure 2. There is no atrophy in the muscles of the right upper extremity of the patient.
Neurological examination revealed muscle strength of $4 / 5$ in the shoulder flexion, extension, and abduction; of $-3 / 5$ in the elbow flexion and extension; of $-3 / 5$ in the wrist flexion and extension; and $-3 / 5$ in the finger abduction and adduction. Biceps, brachioradialis and triceps reflexes were hyperactive. There was no pathological reflex. Sensation examination showed hyperalgesia in all dermatome areas in the right upper extremity.

Laboratory tests (hemogram, sedimentation, CRP, biochemical tests) were normal. Cervical magnetic resonance imaging (MRI) showed no feature except diffuse bulging at the 65 levels $\mathrm{C} 3-\mathrm{C} 4, \mathrm{C} 4-\mathrm{C} 5, \mathrm{C} 5-\mathrm{C} 6$, and C6-C7. There was no root pressure. Brachial plexus MRI 66 showed no space-occupying lesion with a pressure on the brachial plexus. Electroneuromyography (ENMG) showed a low amplitude motor response of the right median nerve, while having no sensation response at digits 1 and 3 . Right ulnar and radial nerve sensation responses were lower in amplitude compared to the left side. Needle electromyograhic analysis showed subacute-chronic neurogenic involvement along with spontaneous denervation potentials in all muscles, with innervated upper-middle-lower truncus (pan-plexopathy), remarkably in muscles innervated by upper and middle truncus in the right brachial plexus. The patient received physical treatment and underwent a rehabilitation program with a hotpack on the right shoulder, transcutaneous electrical nerve stimulation (TENS) on the right upper extremity trace, and neuromuscular electrical stimulation (NMES) on the biceps brachii, extensor carpi radialis longus and brevis muscles. A therapeutic exercise program was given including ROM, stretching, strengthening, improving hand skills. After 20 sessions of this therapeutic program, the patient was re-evaluated. A $50 \%$ reduction was found in visual analogue scale (VAS) score. Neurological examination showed a $1 / 5$ increase in the muscle strength. Increased muscle strength was more remarkable during the follow-up at month 1, with shoulder flexion, extension, and abduction 5/5; elbow flexion and extension 4/5; wrist flexion and extension $4 / 5$; and finger abduction and adduction $4 / 5$.

\section{Discussion}

Differential diagnosis of unilateral acute weakness of the upper extremity may have many etiologies, 
including traumatic injuries (damage to brachial plexus etc.), infectious diseases (polio etc.), allergic collagen autoimmune disorders (acute disseminated encephalomyelitis etc.), biochemical disorders (periodic paralysis etc.), degenerative disorders (multiple sklerosis etc.), anatomical, structural disorders (thoracic outlet syndrome, spinal stenosis etc.) and arteriosclerotic vascular venous disorders (cerebral ischemic attacks etc.) [4]. Approximately $0.5 \%$ to $3 \%$ of patients with herpes zoster infection develops peripheral motor paresis that is a very rare complication [1]. A literature review revealed that in 1991, Ohtake et al. [5] reported a 73-year-old female patient, who developed weakness in the right upper extremity with an axonal degeneration of the proximal portion at level C4-6 following brachial plexus involvement secondary to herpes zoster.

Fabian et al. [3] performed a post-mortem pathological examination after a 78-year-old female patient with monoplegia who developed brachial plexus involvement secondary to herpes zoster died from heart attack, and they detected lymphocytic infiltration and myelin breakdown, but found that axons were preserved. They found perivascular lymphocytic cuffing in the cervical spinal cord, but no anterior horn necrosis. Based on these results, motor paralysis might have been related with an inflammatory process. In 2005, Yoleri et al. [6] reported a case who developed muscle weakness in the shoulder girdle associated with involvement of the C5-7 motor roots and upper truncus of the brachial plexus secondary to herpes zoster. In 2006, Eyigor et al. [7] described a 54-year-old male patient with monoparesis, hyperalgesia, allodynia, edema, and both color and skin-temperature changes, who was diagnosed with complex regional pain syndrome and brachial plexopathy secondary to herpes zoster. Cervical MRI and brachial plexus MRI showed no pathology. Electrophysiological analysis revealed partial degeneration of the superior, medial and inferior truncus in the brachial plexus similar to our case. In 2010 [8], MRI images of a patient with weakness, sensory loss and atrophy due to brachial plexus involvement secondary to herpes zoster showed inflammatory changes in the brachial plexus. Brachial MRI showed no involvement in our patient. In 2011, Alshekhlee et al. [9] described various neuropathic findings, multiple neuropathies, radiculopathy and brachial plexopathy in 3 patients with herpes zoster infection of the upper extremity, and found that weakness and electrophysiological changes were not related with dermatomes. Also in our case no relation was found with dermatomes.

In a study which evaluated 49 patients with zoster associated limb paralysis in 2014, Jones et al. [10] reported that the mean age of onset was 71 years; the duration between rash and weakness was approximately 5.8 days; and $11 \%$ of patients had diabetes mellitus. The mean duration of paralysis was days. Of 22 patients with upper limb paralysis, 4 had multiple cervical level involvement at C5, 6, and 2 at C8, T1, 12, while 2 had ulnar level, and 2 median level involvement. The diagnosis is typically made with ENMG evaluation based on clinical findings. MRI is important for excluding space-occupying lesions involving the brachial plexus and spinal cord, and other pathologies. Imaging is beneficial in eliminating other diagnoses. Often no pathology is determined by imaging methods $[2,10]$. In our case, brachial plexus MRI and cervical MRI also failed to show any pathology to explain the monoparesis.

Our patient developed weakness 3 days after rash, however the duration between skin lesions and development of weakness is variable. In some cases, weakness seems to have developed a few months after the rash. The probability of peripheral axonal damage secondary to sensory and motor involvement and development of complications is often increased in elderly patients and immunocompromised patients [1].

Our case was a 69-year-old patient, and he had concomitant diseases such as hypertension and diabetes mellitus. Functional improvement occurs between 1 and 2 years in most cases [10]. Our patient showed a remarkable improvement pattern 4 months after the onset. Literature shows that use of antivirals at appropriate doses can prevent motor involvement, and use of steroids can also be effective in such cases. Some publications recommend use of iv acyclovir and iv steroids in case of paresis $[1,6]$. We didn't prefer iv steroids due to presence of diabetes mellitus in our patient. Although he received antiviral treatment in early period, he still developed paresis, but it is likely that he might have had more prominent manifestations if he didn't receive such treatment. The patient benefited from the physical treatment, and strengthening rehabilitation program. While he had difficulty in using his hand upper extremity at his presentation, he was able to use it for daily activities very easily during the follow-up. 


\section{Conclusion}

Herpes zoster should be considered in the differential diagnosis of acute monoparesis. It is very important in avoiding unnecessary tests and treatments.

\section{Informed consent}

Written informed consent was obtained from the patient for the publication of this case report.

\section{Conflict of interest}

The authors declared that there are no potential conflicts of interest with respect to the research, authorship, and/or publication of this article.

\section{References}

[1] Rastegar S, Mahdavi SB, Mahmoudi F, Basiri K. Herpes zoster segmental paresis 158 in an immunocompromised breast cancer woman. Adv Biomed Res 2015;4:170.

[2] Ruppert LM, Freeland ML, Stubblefield MD. Segmental zoster paresis of the left upper limb in a pediatric patient. Am J Phys Med Rehabil 2010;89:1024-9.

[3] Fabian VA, Wood B, Crowley P, Kakulas BA. Herpes zoster brachial plexus neuritis. Clin Neuropathol 1997;16:61-4.

[4] Bradley G. W, Daroff B, Fenichel M. G, Jankovic J. Vascular diseases of nervous system. Neurology in Clinical Practice. 4th edn. Vol 1. Elsevier, Butterworth 155 Heinemann, 2004; pp. $343-$ 8.

[5] Ohtake T, Komon T, Hirose K, Tanabe H. Monoparesis due to the brachial plexus 167 neuritis by herpes zoster virus-report of a case. Rinsho Shinkeigaku 1991;31:1245-7.

[6] Yoleri O, Olmez N, Oztura I, Sengul I, Gunaydın R, Memis A. Segmental zoster paresis of the upper extremity: a case report. Arch Phys Med Rehabil 2005;86:1492-4.

[7] Eyigor S, Durmaz B, Karapolat H. Monoparesis with complex regional pain syndrome-like symptoms' due to brachial plexopathy caused by the varicella zoster virus: a case report. Arch Phys Med Rehabil 2006;87:1653-5.

[8] Ayoub T, Raman V, Chowdhury M. Brachial neuritis caused by varicella-zoster diagnosed by changes in barchial plexus on MRI. J Neurol 2010;257:1-4.

[9] Alshekhlee A, Tay E, Buczek M, Shakir ZA, Katirji B. Herpes zoster with motor involvement: discordance between the distributation of skin rash and localization of peripheral nervous system dysfunction. J Clin Neuromuscul Dis 2011;12:153-7. [10] Jones LK, Reda H, Watson JC. Clinical, electrophysiological, and imaging features of zoster-associated limb paresis. Muscle Nerve 2014;50:177-85. 